01

\title{
Вероятности радиационных переходов в спектрах никелеподобных ионов Cd XXI, In XXII, Sn XXIII
}

\author{
(C) А.В. Логинов
}

Петербургский государственный университет путей сообщения Императора Александра I, 190031 Санкт-Петербург, Россия

e-mail: andrlgnv@yandex.ru

Поступила в редакцию 19.09.2018 г.

Полуэмпирически в промежуточной схеме связи выполнен расчет вероятностей электродипольных переходов $3 d^{9} 4 p \rightarrow 3 d^{10}, 3 d^{9} 4 s$ в спектрах никелеподобных ионов Cd XXI, In XXII, Sn XXIII. Радиальные интегралы, необходимые для перехода к абсолютной шкале вероятностей, вычислены с функциями ХартриФока в форме длины.

DOI: $10.21883 /$ OS.2019.02.47191.272-18

\section{Введение}

Судя по библиографии из базы данных [1], спектры никелеподобных ионов - популярный объект для исследования. Эта популярность, в частности, связана с надеждой создать лазер в рентгеновской области спектра на переходе $3 d^{9} 4 d(J=0) \rightarrow 3 d^{9} 4 p(J=1)$ [2]. К настоящему моменту экспериментальные сведения по уровням энергии получены для ионов с зарядом ядра $Z \leq 50$ (т. е. до Sn XXIII) [3], а теоретические сведения как по уровням энергии, так и по радиационным константам - для ионов с $Z \leq 100$ [4-7]. Тем не менее, опубликованных данных по спектрам никелеподобных ионов высоких степеней ионизации, видимо, недостаточно, чтобы выбрать из них „рекомендованные значения“" уровней энергии и вероятностей переходов для заполнения соответствующих столбцов базы данных [1]. Это означает, что продолжение исследований по этой тематике по-прежнему актуально.

В настоящей работе полуэмпирически вычислены вероятности электродипольных переходов $3 d^{9} 4 p \rightarrow 3 d^{10}$, $3 d^{9} 4 s$ в спектрах никелеподобных ионов $\mathrm{Cd} \mathrm{XXI}$, In XXII, Sn XXIII. Отметим, что в упомянутых работах [4-7] расчеты выполнены ab initio. Полагаем, что полуэмпирические данные, полученные здесь на основе высокоточных измерений длин волн переходов, вполне органично дополнят результаты [4-7].

\section{Метод расчета}

Данная работа является непосредственным продолжением работы [8], в которой совершенно аналогичная процедура была выполнена для никелеподобных ионов до $\mathrm{Ag} \mathrm{XX}$ включительно. Расчет проведен в промежуточной схеме связи, при этом угловая часть волновой функции получается диагонализацией матрицы оператора энергии, построенной в $S L$-связи в одноконфигурационном приближении. Радиальные части элементов матрицы оператора энергии рассмат- риваются как параметры, определяемые из предписания наименьших квадратов, сформулированного для собственных значений матрицы оператора энергии и сопоставляемых им экспериментальных уровней энергии. При реализации предписания наименьших квадратов для конфигураций $3 d^{9} 4 l$ рассматривался следующий набор параметров: интегралы Слэтера $F_{d l}^{k}$, $G_{d l}^{k}$ (радиальные интегралы, входящие сомножителями в матричные элементы оператора электростатического взаимодействия), спин-орбитальные константы $\xi_{3 d}$, $\xi_{4 l}$ (радиальные интегралы, входящие сомножителями

Таблица 1. Параметры $\left(\right.$ в $\left.\mathrm{cm}^{-1}\right)$ для конфигурации $3 d^{9} 4 s$ в спектрах Cd XXI, In XXII, Sn XXIII

\begin{tabular}{c|c|c|c}
\hline Параметр & Cd XXI & In XXII & Sn XXIII \\
\hline$F_{d s}^{0}$ & $345907 \pm 57$ & $375300 \pm 67$ & $405779 \pm 43$ \\
$G_{d s}^{2}$ & $30804 \pm 569$ & $31685 \pm 662$ & $32890 \pm 426$ \\
$\xi_{3 d}$ & $23032 \pm 46$ & $25832 \pm 53$ & $28817 \pm 34$ \\
$\Delta E$ & 114 & 132 & 85 \\
$\Delta E^{\prime}$ & 57 & 66 & 43
\end{tabular}

Таблица 2. Параметры (в $\left.\mathrm{cm}^{-1}\right)$ для конфигурации $3 d^{9} 4 p$ в спектрах Cd XXI, In XXII, Sn XXIII

\begin{tabular}{c|c|c|c}
\hline Параметр & Cd XXI & In XXII & Sn XXIII \\
\hline$F_{d p}^{0}$ & $388914 \pm 40$ & $420866 \pm 16$ & $453890 \pm 5$ \\
$F_{d p}^{2}$ & $90350 \pm 600$ & $94307 \pm 270$ & $98369 \pm 69$ \\
$G_{d p}^{1}$ & $28530 \pm 181$ & $30107 \pm 82$ & $31225 \pm 24$ \\
$G_{d p}^{3}$ & $29334 \pm 685$ & $30762 \pm 371$ & $31791 \pm 78$ \\
$\xi_{3 d}$ & $23009 \pm 31$ & $25818 \pm 18$ & $28783 \pm 3$ \\
$\xi_{4 p}$ & $52811 \pm 139$ & $59195 \pm 41$ & $66576 \pm 7$ \\
$F_{1}$ & $-106 \pm 23$ & $-158 \pm 12$ & $-164 \pm 3$ \\
$G_{2}$ & $-40 \pm 39$ & - & $-47 \pm 5$ \\
$T_{111}$ & $3431 \pm 1160$ & $955 \pm 440$ & - \\
$\Delta E$ & 98 & 56 & 11 \\
$\Delta E^{\prime}$ & 42 & 25 & 4
\end{tabular}


Таблица 3. Длины волн $(\lambda, \mathrm{nm})$ и вероятности $\left(A, \mathrm{~s}^{-1}\right)$ переходов $3 d^{9} 4 p \rightarrow 3 d^{9} 4 s$ в спектрах Cd XXI, In XXII, Sn XXIII

\begin{tabular}{|c|c|c|c|c|c|c|c|}
\hline \multirow{2}{*}{$\begin{array}{c}\text { Переход } \\
3 d^{9} 4 p \rightarrow 3 d^{9} 4 s\end{array}$} & \multicolumn{3}{|c|}{ Cd XXI } & \multicolumn{2}{|c|}{ In XXII } & \multicolumn{2}{|c|}{ Sn XXIII } \\
\hline & $\lambda$ & $A$ & $A[5]$ & $\lambda$ & $A$ & $\lambda$ & $A$ \\
\hline $01 \rightarrow 11$ & 25.311 & $2.47+10$ & $2.20+10$ & 23.874 & $2.76+10$ & 22.488 & $3.09+10$ \\
\hline $11 \rightarrow 11$ & 29.474 & $3.81+8$ & & 28.141 & $5.18+8$ & 26.887 & $7.19+8$ \\
\hline $11 \rightarrow 21$ & 25.633 & $7.01+9$ & $6.84+9$ & 24.216 & $6.98+9$ & 22.901 & $6.62+9$ \\
\hline $11 \rightarrow 22$ & 29.967 & $1.02+10$ & $0.85+10$ & 28.598 & $1.13+10$ & 27.317 & $1.27+10$ \\
\hline $12 \rightarrow 11$ & 27.363 & $2.37+9$ & $2.12+9$ & 26.034 & $2.41+9$ & 24.790 & $2.35+9$ \\
\hline $12 \rightarrow 21$ & 24.022 & $1.91+10$ & $1.59+10$ & 22.640 & $2.24+10$ & 21.361 & $2.65+10$ \\
\hline $12 \rightarrow 22$ & 27.788 & $4.09+9$ & $4.02+9$ & 26.425 & $3.92+9$ & 25.155 & $3.57+9$ \\
\hline $13 \rightarrow 11$ & 24.011 & $2.48+10$ & $2.16+10$ & 22.643 & $2.76+10$ & 21.355 & $3.10+10$ \\
\hline $13 \rightarrow 21$ & 21.399 & $1.88+9$ & & 20.031 & $1.97+9$ & 18.761 & $1.99+9$ \\
\hline $13 \rightarrow 22$ & 24.337 & $2.77+9$ & & 22.938 & $3.17+9$ & 21.625 & $3.63+9$ \\
\hline $21 \rightarrow 11$ & 36.709 & $8.98+6$ & & 35.585 & $8.30+6$ & 34.556 & $7.60+6$ \\
\hline $21 \rightarrow 21$ & 30.936 & $1.13+9$ & & 29.533 & $1.33+9$ & 28.239 & $1.56+9$ \\
\hline $21 \rightarrow 22$ & 37.477 & $5.88+7$ & & 36.319 & $5.26+7$ & 35.270 & $4.79+7$ \\
\hline $21 \rightarrow 31$ & 30.304 & $1.31+10$ & $1.13+10$ & 28.935 & $1.39+10$ & 27.668 & $1.48+10$ \\
\hline $22 \rightarrow 11$ & 30.126 & $7.90+9$ & $6.57+9$ & 28.777 & $8.45+9$ & 27.534 & $9.04+9$ \\
\hline $22 \rightarrow 21$ & 26.125 & $1.95+9$ & $1.76+9$ & 24.686 & $1.91+9$ & 23.368 & $1.82+9$ \\
\hline $22 \rightarrow 22$ & 30.641 & $5.18+9$ & $4.34+9$ & 29.255 & $5.73+9$ & 27.985 & $6.31+9$ \\
\hline $22 \rightarrow 31$ & 25.673 & $8.19+7$ & & 24.267 & $9.22+7$ & 22.976 & $9.63+7$ \\
\hline $23 \rightarrow 11$ & 27.694 & $8.72+8$ & & 26.348 & $8.49+8$ & 25.075 & $7.92+8$ \\
\hline $23 \rightarrow 21$ & 24.276 & $2.32+10$ & $2.05+10$ & 22.877 & $2.60+10$ & 21.573 & $2.91+10$ \\
\hline $23 \rightarrow 22$ & 28.128 & $7.87+8$ & & 26.748 & $7.50+8$ & 25.448 & $7.08+8$ \\
\hline $23 \rightarrow 31$ & 23.885 & $2.43+9$ & & 22.517 & $2.97+9$ & 21.238 & $3.61+9$ \\
\hline $24 \rightarrow 11$ & 23.547 & $1.27+10$ & $1.09+10$ & 22.193 & $1.44+10$ & 20.927 & $1.64+10$ \\
\hline $24 \rightarrow 21$ & 21.029 & $9.61+7$ & & 19.678 & $8.66+7$ & 18.430 & $8.69+7$ \\
\hline $24 \rightarrow 22$ & 23.860 & $1.71+10$ & $1.49+10$ & 22.476 & $1.89+10$ & 21.186 & $2.10+10$ \\
\hline $24 \rightarrow 31$ & 20.735 & $2.75+8$ & & 19.411 & $2.77+8$ & 18.185 & $2.79+8$ \\
\hline $31 \rightarrow 21$ & 30.271 & $8.94+9$ & $7.54+9$ & 28.921 & $9.52+9$ & 27.677 & $1.01+10$ \\
\hline $31 \rightarrow 22$ & 36.507 & $1.03+7$ & & 35.398 & $8.49+6$ & 34.399 & $7.49+6$ \\
\hline $31 \rightarrow 31$ & 29.666 & $5.85+9$ & $4.86+9$ & 28.348 & $6.33+9$ & 27.129 & $6.84+9$ \\
\hline $32 \rightarrow 21$ & 23.786 & $1.11+10$ & $9.48+9$ & 22.410 & $1.26+10$ & 21.122 & $1.43+10$ \\
\hline $32 \rightarrow 22$ & 27.473 & $2.25+8$ & & 26.112 & $2.04+8$ & 24.823 & $1.92+8$ \\
\hline $32 \rightarrow 31$ & 23.411 & $1.92+10$ & $1.66+10$ & 22.064 & $2.14+10$ & 20.801 & $2.38+10$ \\
\hline $33 \rightarrow 21$ & 21.514 & $3.58+8$ & & 20.117 & $3.39+8$ & 18.829 & $3.37+8$ \\
\hline $33 \rightarrow 22$ & 24.487 & $2.70+10$ & $2.30+10$ & 23.051 & $3.03+10$ & 21.716 & $3.40+10$ \\
\hline $33 \rightarrow 31$ & 21.207 & $1.68+8$ & & 19.838 & $1.59+8$ & 18.573 & $1.56+8$ \\
\hline $41 \rightarrow 31$ & 24.525 & $2.72+10$ & $2.31+10$ & 23.092 & $3.04+10$ & 21.751 & $3.41+10$ \\
\hline
\end{tabular}

Примечание. Значения вероятностей переходов приведены в следующей форме: например, $2.72+10=2.72 \cdot 10^{10}$.

Таблица 4. Длины волн $(\lambda, \mathrm{nm})$ и вероятности $\left(A, \mathrm{~s}^{-1}\right)$ переходов $3 d^{9} 4 p \rightarrow 3 d^{10}$ в спектрах Cd XXI, In XXII, Sn XXIII

\begin{tabular}{c|c|c|c|c|c|c|c|c|c}
\hline \multirow{2}{*}{$\begin{array}{c}\text { Переход } \\
3 d^{9} 4 p \rightarrow 3 d^{10}\end{array}$} & \multicolumn{3}{|c|}{ Cd XXI } & \multicolumn{3}{c|}{ In XXII } & \multicolumn{3}{c}{ Sn XXIII } \\
\cline { 2 - 10 } & $\lambda$ & $A$ & $A[6]$ & $\lambda$ & $A$ & $A[6]$ & $\lambda$ & $A$ & $A[6]$ \\
\hline $11 \rightarrow 01$ & 2.893 & $4.59+10$ & $5.10+10$ & 2.674 & $6.37+10$ & $7.97+10$ & 2.479 & $9.43+10$ & $1.22+11$ \\
$12 \rightarrow 01$ & 2.872 & $8.93+11$ & $1.21+12$ & 2.653 & $1.01+12$ & $1.39+12$ & 2.460 & $1.13+12$ & $1.57+12$ \\
$13 \rightarrow 01$ & 2.830 & $1.61+11$ & $1.93+11$ & 2.613 & $1.80+11$ & $2.14+11$ & 2.421 & $1.97+11$ & $2.40+11$
\end{tabular}

в матричные элементы оператора спин-орбитального взаимодействия), эффективные параметры $F_{k}, G_{k}$, которые принято называть интегралами Слэтера с запрещенными рангами, магнитные эффективные параметры $T_{k_{1} k_{2} k_{3}}$ (для конфигураций $3 d^{9} 4 p$ ). Угловые коэффициенты для интегралов Слэтера и спин-орбитальных констант вычислялись по общеизвестным формулам (например, [9]), детали вычислений угловых коэффициентов для эффективных параметров можно найти в [8].

Качество реализации предписания наименьших квадратов определяется дисперсиями параметров, а также 
стандартными $(\Delta E)$ и среднеквадратичными $\left(\Delta E^{\prime}\right)$ отклонениями по энергии:

$$
\begin{gathered}
\Delta E=\sqrt{\sum_{i=1}^{n}\left(E_{\text {calc }}^{i}-E_{\text {exp }}^{i}\right)^{2} /(n-m)}, \\
\Delta E^{\prime}=\sqrt{\sum_{i=1}^{n}\left(E_{\text {calc }}^{i}-E_{\text {exp }}^{i}\right)^{2} / n},
\end{gathered}
$$

где $n$ - число экспериментальных уровней, включенных в вычислительную процедуру, $m$ - число свободно варьируемых параметров, $E_{\text {calc }}^{i}, E_{\text {exp }}^{i}-$ соответственно вычисленное и экспериментальное значения энергии $i$ го уровня.

\section{Результаты}

\section{Параметры}

Таблицы 1, 2 содержат значения параметров, их дисперсии и среднеквадратичные отклонения $\Delta E, \Delta E^{\prime}$, полученные из предписания наименьших квадратов для конфигураций $3 d^{9} 4 s$ (табл. 1) и $3 d^{9} 4 p$ (табл. 2). Экспериментальные уровни энергии были взяты из [3]. Заметим, что в статье [3] допущена опечатка в той части Table II, где приведены значения уровней энергии конфигурации $3 d^{9} 4 p$ для $\mathrm{Cd} \mathrm{XXI}$. Эти же значения (без опечатки) повторно опубликованы в работе [10].

Из табл. 1,2 следует, что интегралы Слэтера и спинорбитальные константы определены очень хорошо - с точностью до 3-5 значащих цифр. Что касается эффективных параметров $\left(F_{1}, G_{2}, T_{111}\right)$, то они определены, скажем так, вполне приемлемо. При этом отметим, что в [3] для трех уровней энергии конфигурации $3 d^{9} 4 p$ (из 12 возможных) отсутствуют экспериментальные значения в случае Sn XXIII, а экспериментальные значения этих же уровней для In XXII отмечены вопросительным знаком (авторы сомневаются в их надежности). Поэтому при реализации предписания наименьших квадратов для конфигураций $3 d^{9} 4 p$ In XXII, Sn XXIII упомянутые уровни были исключены из вычислительной процедуры. По этой причине не все эффективные параметры (из трех рассмотренных) оказались существенными для In XXII и Sn XXIII.

В целом, привлекая результаты, полученные в [8] для предыдущих членов изоэлектронной последовательности, можно заключить, что параметры монотонно изменяются с ростом $Z$ (исключая скачок эффективного параметра $T_{111}$ для In XXII). Это обстоятельство наряду с небольшими значениями среднеквадратичных отклонений $\Delta E, \Delta E^{\prime}$ свидетельствует об адекватности реализованной процедуры наименьших квадратов.

\section{Вероятности переходов}

Волновые функции промежуточной связи, полученные в предыдущем разделе, были использованы для расчета
Таблица 5. Времена жизни (в ps) уровней $3 d^{9} 4 p$ в спектрах Cd XXI, In XXII, Sn XXIII

\begin{tabular}{l|c|c|c}
\hline \multicolumn{1}{c|}{ Уровень } & Cd XXI & In XXII & Sn XXIII \\
\hline $01\left({ }^{3} P_{0}\right)$ & 40.4 & 36.2 & 32.4 \\
$11\left({ }^{3} P_{1}\right)$ & 15.8 & 12.1 & 8.74 \\
$12\left({ }^{1} P_{1}\right)$ & 1.09 & 0.962 & 0.862 \\
$13\left({ }^{3} D_{1}\right)$ & 5.26 & 4.77 & 4.27 \\
$21\left({ }^{3} P_{2}\right)$ & 70.0 & 65.2 & 60.9 \\
$22\left({ }^{3} F_{2}\right)$ & 66.2 & 61.8 & 57.9 \\
$23\left({ }^{1} D_{2}\right)$ & 36.6 & 32.7 & 29.2 \\
$24\left({ }^{3} D_{2}\right)$ & 33.2 & 29.7 & 26.5 \\
$31\left({ }^{3} F_{3}+{ }^{1} F_{3}\right)$ & 67.6 & 63.0 & 58.9 \\
$32\left({ }^{3} D_{3}\right)$ & 32.7 & 29.3 & 26.2 \\
$33\left({ }^{3} F_{3}+{ }^{1} F_{3}\right)$ & 36.4 & 32.5 & 29.0 \\
$41\left({ }^{3} F_{4}\right)$ & 36.8 & 32.8 & 29.3
\end{tabular}

Примечание. В скобках указана $S L$-компонента волновой функции с максимальным весом.

вероятностей электродипольных радиационных переходов $3 d^{9} 4 p \rightarrow 3 d^{10}, 3 d^{9} 4 s$. Для перехода к абсолютной шкале вероятностей были использованы радиальные интегралы переходов, рассчитанные в форме длины с функциями Хартри-Фока по программе [11]. В табл. 3,4 приведены длины волн и вероятности указанных переходов. Уровни в этих таблицах обозначаются двумя цифрами. Первая цифра дает значение полного момента $J$ для данного уровня, вторая - порядковый номер данного уровня среди совокупности уровней с указанным значением $J$, упорядоченных по возрастанию энергии. В табл. 5 даны времена жизни уровней $3 d^{9} 4 p$, полученные суммированием вероятностей переходов $k \rightarrow i$ из табл. 3, 4:

$$
\tau_{k}=1 / \sum_{i} A_{k i}
$$

Для сравнения в табл. 3,4 приведены те данные работ $[5,6]$, которые можно непосредственно сопоставить нашим результатам. Данные $[5,6]$ получены на основе „relativistic many-body perturbation theory (RMBPT)“، Легко видеть, что они вполне приемлемо согласуются с данными настоящей работы. При этом значения вероятностей переходов $3 d^{9} 4 p \rightarrow 3 d^{10}$ из [6] (табл. 4) систематически превосходят наши, а значения вероятностей переходов $3 d^{9} 4 p \rightarrow 3 d^{9} 4 s$ из [5] (табл. 3), напротив, систематически меньше таковых. Это означает, что расхождение с результатами $[5,6]$ можно описать масштабирующими множителями и отнести их, например, на счет различия в радиальных интегралах переходов. А в целом можно заключить, что $a b$ initio метод RMBPT $[5,6]$ и полуэмпирическая вычислительная процедура из настоящей работы привели в данном случае к очень похожим результатам. Сопоставление с экспериментальными радиационными константами невозможно ввиду их отсутствия в литературе. 


\section{Список литературы}

[1] Kramida A., Ralchenko Yu., Reader J., and NIST ASD Team. NIST Atomic Spectra Database (ver. 5.3).Электронный ресурс. Режим доступа: http://physics.nist.gov/asd

[2] Chen B.K. et al. // Appl. Phys. B. V. 106. N 4. P. 817.

[3] Churilov S.S., Ryabtsev A.N. // Phys. Scr. 1988. V. 38. P. 326.

[4] Иванова Е.П. // Опт. и спектр. 2015. Т. 118. № 4. С. 535.

[5] Safronova U.I., Safronova A.S., Beiersdorfer P. // J. Phys. B. 2007. V. 40. P. 955.

[6] Safronova U.I., Safronova A.S., Hamasha S.M., Beiersdorfer P. // At. Dat. Nucl. Dat. Tabl. 2006. V. 92. P. 47.

[7] Safronova U.I., Safronova A.S., Beiersdorfer P. // Phys. Rev. A. 2008. V. 77. P. 032506.

[8] Логинов А.В. // Опт. и спектр. 2015. Т. 118. № 3. С. 355.

[9] Wybourne B.G. Spectroscopic Properties of the Rare Earths. N.Y:: Wiley, 1965.

[10] Rahman A., Hammarsten E.C., Sakadzic S., Rocca J.J., Wyart J.-F. // Phys. Scr. 2003. V. 67. P. 414.

[11] Cowan R.D. The Theory of Atomic Structure and Spectra. Berkeley: Univ. Calif. Press, 1981. Cowan computer codes. 